\title{
Fast pulsed operation of a small non- radioactive electron source with continuous emission current control
}

Cite as: Rev. Sci. Instrum. 86, 065102 (2015); https://doi.org/10.1063/1.4921707

Submitted: 25 February 2015. Accepted: 13 May 2015. Published Online: 05 June 2015

P. Cochems, A. T. Kirk, E. Bunert, M. Runge, P. Goncalves, and S. Zimmermann
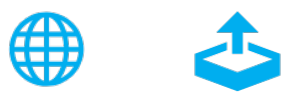

View Online

\section{ARTICLES YOU MAY BE INTERESTED IN}

Shutterless ion mobility spectrometer with fast pulsed electron source

Review of Scientific Instruments 88, 024102 (2017); https://doi.org/10.1063/1.4976021

A compact high-resolution X-ray ion mobility spectrometer

Review of Scientific Instruments 87, 053120 (2016); https://doi.org/10.1063/1.4950866

In-circuit-measurement of parasitic elements in high gain high bandwidth low noise transimpedance amplifiers

Review of Scientific Instruments 85, 124703 (2014); https://doi.org/10.1063/1.4902854

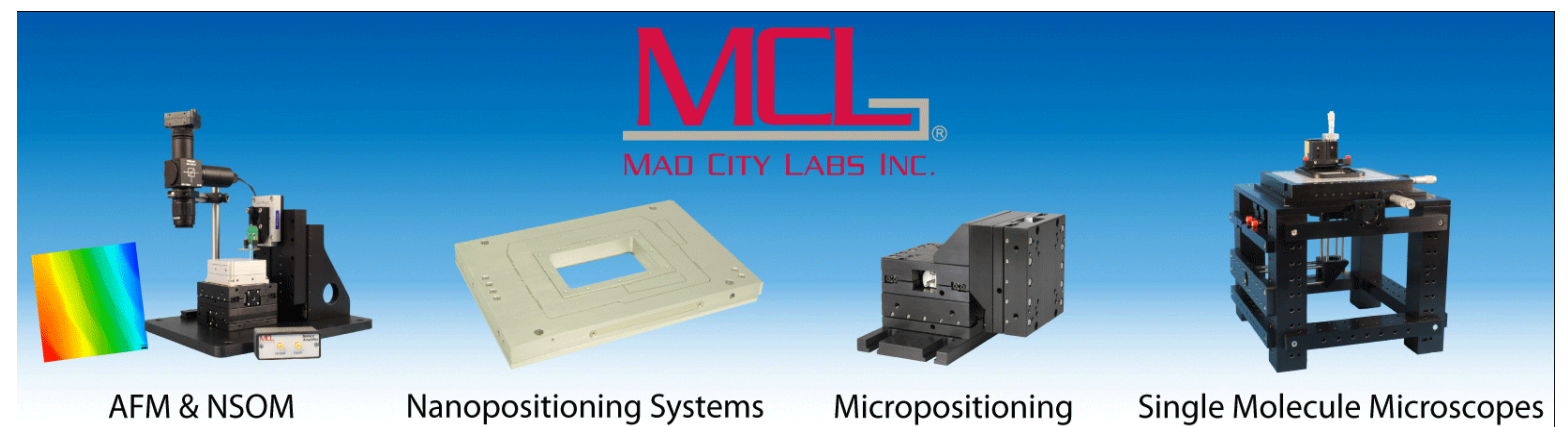




\title{
Fast pulsed operation of a small non-radioactive electron source with continuous emission current control
}

\author{
P. Cochems, A. T. Kirk, E. Bunert, M. Runge, P. Goncalves, and S. Zimmermann \\ Department of Sensors and Measurement Technology, Institute of Electrical Engineering and Measurement \\ Technology, Leibniz University Hannover, Hannover, Germany
}

(Received 25 February 2015; accepted 13 May 2015; published online 5 June 2015)

\begin{abstract}
Non-radioactive electron sources are of great interest in any application requiring the emission of electrons at atmospheric pressure, as they offer better control over emission parameters than radioactive electron sources and are not subject to legal restrictions. Recently, we published a simple electron source consisting only of a vacuum housing, a filament, and a single control grid. In this paper, we present improved control electronics that utilize this control grid in order to focus and defocus the electron beam, thus pulsing the electron emission at atmospheric pressure. This allows short emission pulses and excellent stability of the emitted electron current due to continuous control, both during pulsed and continuous operations. As an application example, this electron source is coupled to an ion mobility spectrometer. Here, the pulsed electron source allows experiments on gas phase ion chemistry (e.g., ion generation and recombination kinetics) and can even remove the need for a traditional ion shutter. (C) 2015 AIP Publishing LLC. [http://dx.doi.org/10.1063/1.4921707]
\end{abstract}

\section{INTRODUCTION}

The emission of electrons at atmospheric pressure is necessary in a wide variety of sensor applications, for example, in both ion mobility spectrometry (IMS) $)^{1,2}$ and mass spectrometry (MS) ${ }^{3,4}$ utilizing atmospheric pressure chemical ionization (APCI). Here, high energetic electrons initiate the chemical ionization process, which is described in detail in Refs. 1 and 5. Other applications include further sensors principles based on analyte ionization, such as electron capture devices ${ }^{6,7}$ or smoke detectors. ${ }^{8,9}$ In most cases, a radioactive beta minus decay, e.g., from ${ }^{3} \mathrm{H}$ or ${ }^{63} \mathrm{Ni}$, is used as the electron source. Radioactive electron sources do not require maintenance, electrical power, or additional instrumentation to operate and are therefore a convenient choice for many applications. However, non-radioactive electron sources offer several distinct advantages over their radioactive counterparts and are therefore of major interest in many of the mentioned applications. First, radioactive sources are subject to legal restrictions and cannot be used in many applications. Second, the ionization parameters of a radioactive electron source are defined by the choice of material and activity. A non-radioactive electron source can however be controlled in order to vary ionization parameters such as ionization time, kinetic electron energy, electron current, and density. Analytical control over these parameters offers an additional separation dimension. For example, the ability to generate short electron pulses offers the ability to investigate the gas phase reaction kinetics of ion generation and ion-ion recombination, which can be used to improve the separation power of ion mobility spectrometers. ${ }^{10,11}$ However, in order to maintain comparability with data acquired from radioactive sources, the non-radioactive electron source should be able to emit electrons within a similar energy range. Electrons emitted by ${ }^{3} \mathrm{H}$ have a maximum kinetic energy of $18.6 \mathrm{keV}$ and an average kinetic energy of $5.7 \mathrm{keV},{ }^{12}$ while ${ }^{63} \mathrm{Ni}$ emits faster electrons with a maximum kinetic energy of $67 \mathrm{keV}$ and an average kinetic energy of $17 \mathrm{keV} .{ }^{13} \mathrm{~A}$ widely used and wellunderstood method to generate free electrons is thermionic emission in vacuum. ${ }^{14} \mathrm{~A}$ thermionic emitter (filament) is heated to stimulate electrons in the solid state and an electric field provides additional energy to the electrons. Eventually, electrons overcome the work function and can leave the solid state. In order to reach kinetic electron energies of a few $\mathrm{keV}$, a vacuum chamber is required to avoid electrical breakdown and collisions with residual gas. At the end of the evacuated acceleration region, an electron transfer from vacuum to atmospheric pressure is necessary.

In Refs. 10 and 15, such a non-radioactive electron source primarily designed for the excitation of a vacuum ultraviolet (VUV) light source is presented. This electron source generates free electrons from a thermionic emitter in a glass vacuum tube. The free electrons are accelerated to $8-12 \mathrm{keV}$ and focused onto a $300 \mathrm{~nm}$ thin electron transparent $\mathrm{Si}_{3} \mathrm{~N}_{4}$-membrane. One drawback of this setup is the complex electron focusing system using an electrostatic lens system and a magnetic electron beam guide. Both the lens system and the magnetic electron beam guide require an extended vacuum chamber leading to equally large outer dimensions. Another disadvantage is the method used for pulsed electron emission. As the electron beam is completely blanked between two ionization pulses, the emission current is only measurable during the ionization pulse. However, the ionization time in ion mobility spectrometry applications can be as short as $1 \mu \mathrm{s}$ with electron currents of less than $1 \mathrm{nA}$. In order to directly measure such a current pulse, the employed transimpedance amplifier would need a bandwidth above $1 \mathrm{MHz}$, a gain of about $1 \mathrm{G} \Omega$, and a noise current significantly below $100 \mathrm{pA}$. These requirements seem impossible to meet. Thus, the electron source presented in Refs. 10 and 15 uses a relatively slow current amplifier and measures the average emission current over time. From the known duty cycle, the emission 


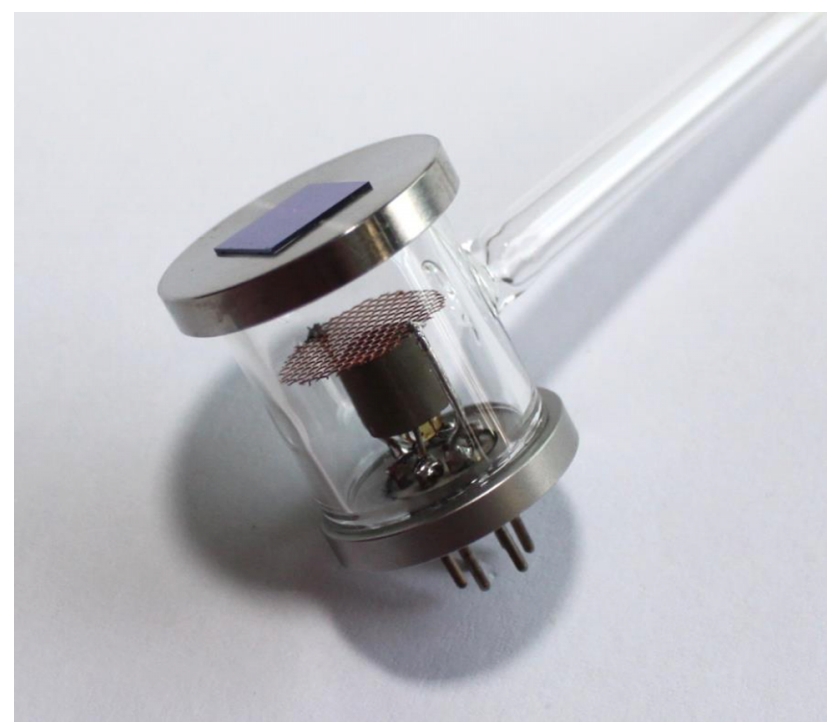

FIG. 1. Photo of the small electron source.

current during the actual emission is then calculated. However, as the duration of an ion mobility spectrum is in the range of about $10 \mathrm{~ms}$, the average emission current can be as much as 10000 times lower than the actual emission current. This reduction of the signal results in a reduction of the signal-tonoise ratio of the measurement by about $80 \mathrm{~dB}$ and requires a slow control system, leading to an extremely slow response.

In order to avoid most of the problems mentioned above and to miniaturize the setup, we developed a highly compact electron source concept employing only a single control grid as published in Ref. 16. This new setup primarily consists of a small, evacuated glass tube with the necessary electrical feedthroughs on the bottom side and a metal electrode on the top side as shown in Figures 1 and 2.

The glass evacuation port is sealed off and the top metal electrode with a hole of $1 \mathrm{~mm}$ in diameter at its center serves as an acceleration electrode. This hole is sealed with a $300 \mathrm{~nm}$ thin $\mathrm{Si}_{3} \mathrm{~N}_{4}$ membrane, which separates the vacuum

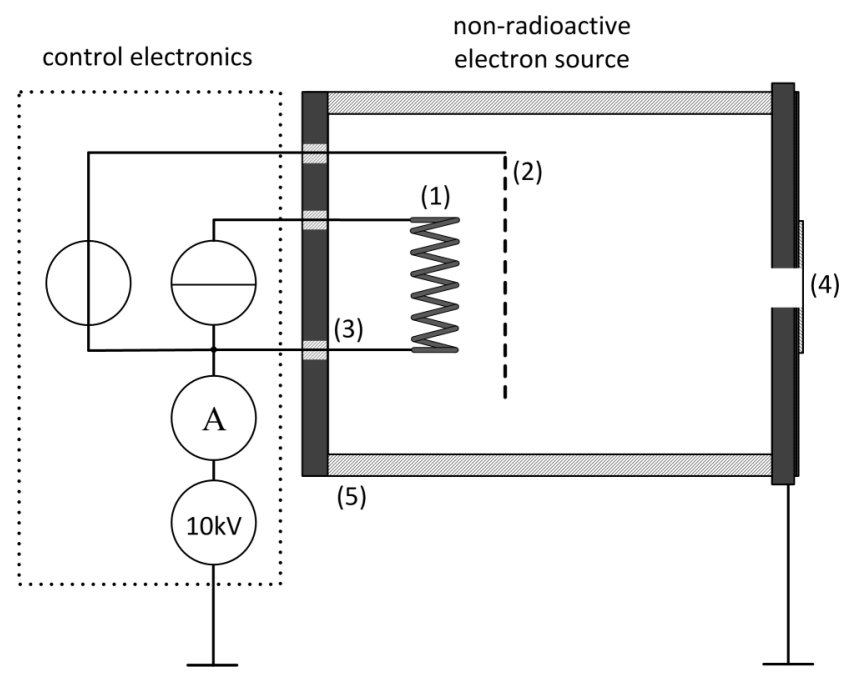

FIG. 2. Electron source setup: (1) filament, (2) control grid, (3) vacuum feedthrough, (4) $\mathrm{Si}_{3} \mathrm{~N}_{4}$ membrane, and (5) glass housing. inside the electron source from the atmospheric pressure but is transparent to electrons with a kinetic energy of at least $8 \mathrm{keV} .^{17,18}$ Inside the glass tube, a tungsten filament is installed, which is electrically heated in order to stimulate thermionic emission of electrons. Above the filament, the single control grid is mounted, which was designed in Ref. 16 to control the thermionic emission current by varying the field strength at the surface of the filament. Both the filament and the control grid are referenced to a potential of $-10 \mathrm{kV}$ relative to the acceleration electrode. The electric field generated by this potential difference accelerates the emitted electrons to a kinetic energy of $10 \mathrm{keV}$, allowing them to pass through the $\mathrm{Si}_{3} \mathrm{~N}_{4}$ membrane and transmit to atmospheric pressure. A getter, which is not shown in the schematic drawings in Figure 2 but visible in Figure 1, is used to maintain the vacuum inside the sealed vacuum chamber.

\section{METHOD FOR PULSED OPERATION}

While the electron source presented in Ref. 16 already offers the advantage of non-radioactive electron emission with controllable electron energy and electron current, it still lacks the ability to produce a pulsed electron beam. This pulsed operation however is crucial for several of the applications described in the Introduction, such as experiments on gas phase ion chemistry, and can therefore be considered as a key element in the development of a non-radioactive electron source. Furthermore, it should not interfere with the control of the electron current emitted at atmospheric pressure, as the methods described in the Introduction do when controlling extremely low electron currents. A radioactive tritium source with an activity of $300 \mathrm{MBq}$ emits an electron current of about $50 \mathrm{pA}$, which needs to be reproducible using a nonradioactive electron source. Due to the missing electron beam focusing in our electron source design, the electron beam is significantly larger than the small hole in the accelerating electrode and only a fraction of the electron beam can actually pass through the small hole in front of the $\mathrm{Si}_{3} \mathrm{~N}_{4}$ membrane. Thus, the interior emission current, which is the electron current emitted from the filament/control grid combination, will also be significantly larger than the exterior emission current, which is the electron current observed at atmospheric pressure observed outside the source. This damping factor between the interior emission current and the exterior emission current is about 20 in our setup when the emission is switched on based on the diameter of the partially focused electron beam compared to the diameter of the hole in the acceleration electrode. As only small exterior emission currents are desired, this damping allows for controlling a significantly larger interior emission current and therefore offers several advantages. First, a current of a few nA can be controlled more easily than a current of a few pA when using a tungsten filament. Second, this damping improves the signal-to-noise ratio of the current measurement by $26 \mathrm{~dB}$ in a constant noise environment. As the thermionic emission current of the filament in the $\mathrm{nA}$ range is still very small for a thermionic emitter, a very long lifetime of the tungsten filament can be expected despite this attenuation. In fact, too cold filaments may even result in reduced lifetimes of the 

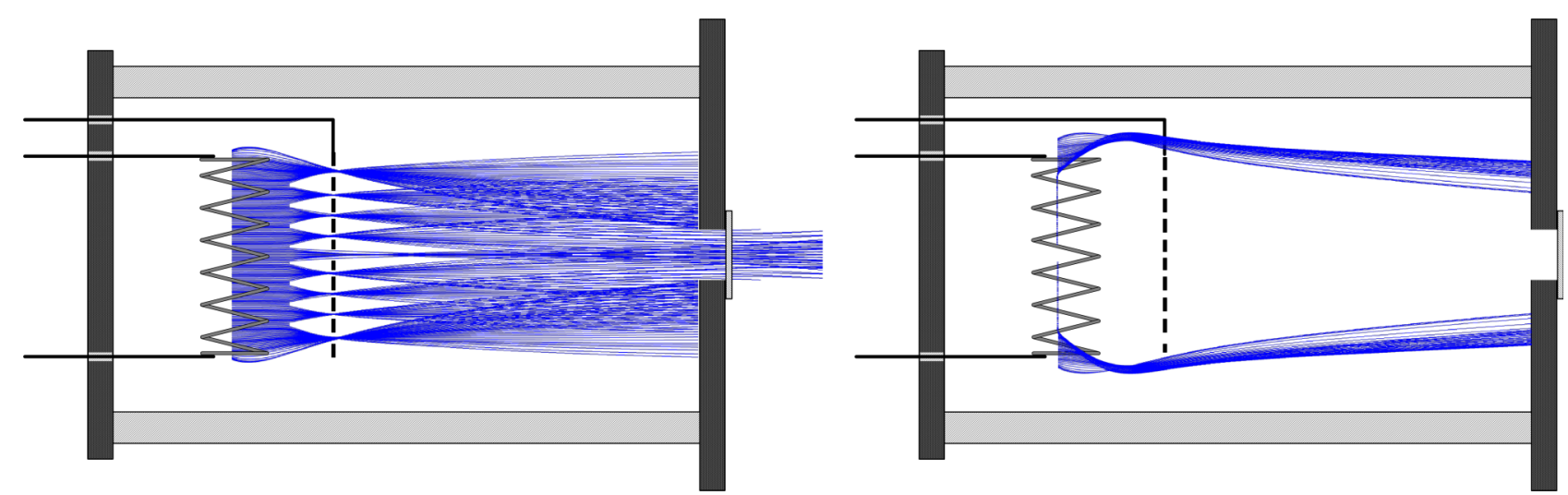

FIG. 3. COMSOL Multiphysics simulation of the partially focused (left) and unfocussed (right) electron trajectories achieved by two different control grid voltages.

emitter due to increasing pressure inside the vacuum chamber, as the filament temperature is an important parameter in some gettering processes. ${ }^{19}$

The damping ratio depends on the shape of the electric field inside the non-radioactive electron source, as perturbations in the field shape can deflect the electrons and therefore focus or defocus the electron beam. Thus, the control grid above the filament can not only be used to control the exterior emission current by controlling the extraction of electrons from the filament as in Ref. 16 but also by shaping the electric field inside the electron source as shown by the COMSOL Multiphysics simulation in Figure 3. This simulation is based on a simple rotationally symmetrical model using the electric potentials given in the Introduction and generates electrons without initial kinetic energy at the filament. Their trajectories are then calculated by the particle tracing module.

If the thermionic electron emission is operated in saturation, meaning that all thermionically emitted electrons are extracted by the existing electric field, the interior emission current will remain constant independent of the voltage applied to the control grid. Only the exterior emission current will vary depending on whether the voltage applied to the control grid focusses or defocusses the electron beam. By switching between two voltages with different damping factors, one partially focusing electrons onto the $\mathrm{Si}_{3} \mathrm{~N}_{4}$ membrane and one completely defocussing the electron beam, the exterior emission current can therefore be switched on and off. As the damping factor for a certain voltage is constant, it can be easily obtained from a static measurement.

The novel pulsing method is based on exactly this effect, as the voltage of the control grid can be switched easily and quickly using suitable electronics. In fact, as shown in Figure 8 in the section titled Experimental, the required voltage difference between partially focusing and defocussing the electron beam can be as low as $10 \mathrm{~V}$. Thus, this method is well suited for fast pulsed operation with low instrumental effort. As the interior emission current stays constant while the exterior emission current is pulsed, it can be continuously measured and regulated by adjusting the filament heating power. Due to the continuous measurement of the interior emission current, this method has a significantly improved signal-to-noise ratio compared to commercially available electron sources that use an averaging method and correct for the duty cycle as described in the Introduction. In combination with the increased interior emission current, this will result in an improvement of the signal-to-noise ratio in the ion mobility spectrometer application above by about $100 \mathrm{~dB}$ - five orders of magnitude. Thus, it is now possible to control the exterior emission current observed at atmospheric pressure precisely, even during extremely short emission pulses. Furthermore, a constant and reproducible exterior emission current can be achieved in a very short time, even for extremely low values.

\section{ELECTRONIC CONCEPT}

In order to operate our electron source, control electronics providing the acceleration voltage, the filament heating current, and the control grid voltage as well as measuring the emission current are necessary. The basic setup is shown in Figure 4. The electronics have been developed with the goal of providing a flexible platform for controlling different thermionic emitter setups during their optimization. However, it was taken care to use no special components in order to keep the electronic inexpensive and simple for further use of the small electron source in low-cost applications.

The "isolated power supply" represents the electrically isolated power supply for the electronics at high voltage potential. At the time of construction, no commercially available DC/DC converter able to isolate at least $16 \mathrm{kV}$ and to provide $12 \mathrm{~W}$ output power as well as a sufficiently high isolation resistance was known to us. These specifications arise from the necessary acceleration voltage when coupled to an ion mobility spectrometer, the necessary power to heat larger tungsten filaments not used in this work and the allowable leakage current. In order to measure currents in the order of a single nA, the DC/DC converter would require an insulation resistance of at least $16 \mathrm{~T} \Omega$ to limit the offset of the current measurement to the measuring range. To ensure such large insulation resistance and high isolation voltage, a transformer was wound satisfying these particular requirements. The output voltage feedback is implemented using an optical fiber, in order to ensure the insulation.

The "high voltage source" is the power supply that generates the acceleration voltage, which needs to be adjustable in the range of $-4 \mathrm{kV}$ to $-16 \mathrm{kV}$ in our application. The stability of this voltage is crucial for the current measurement, 


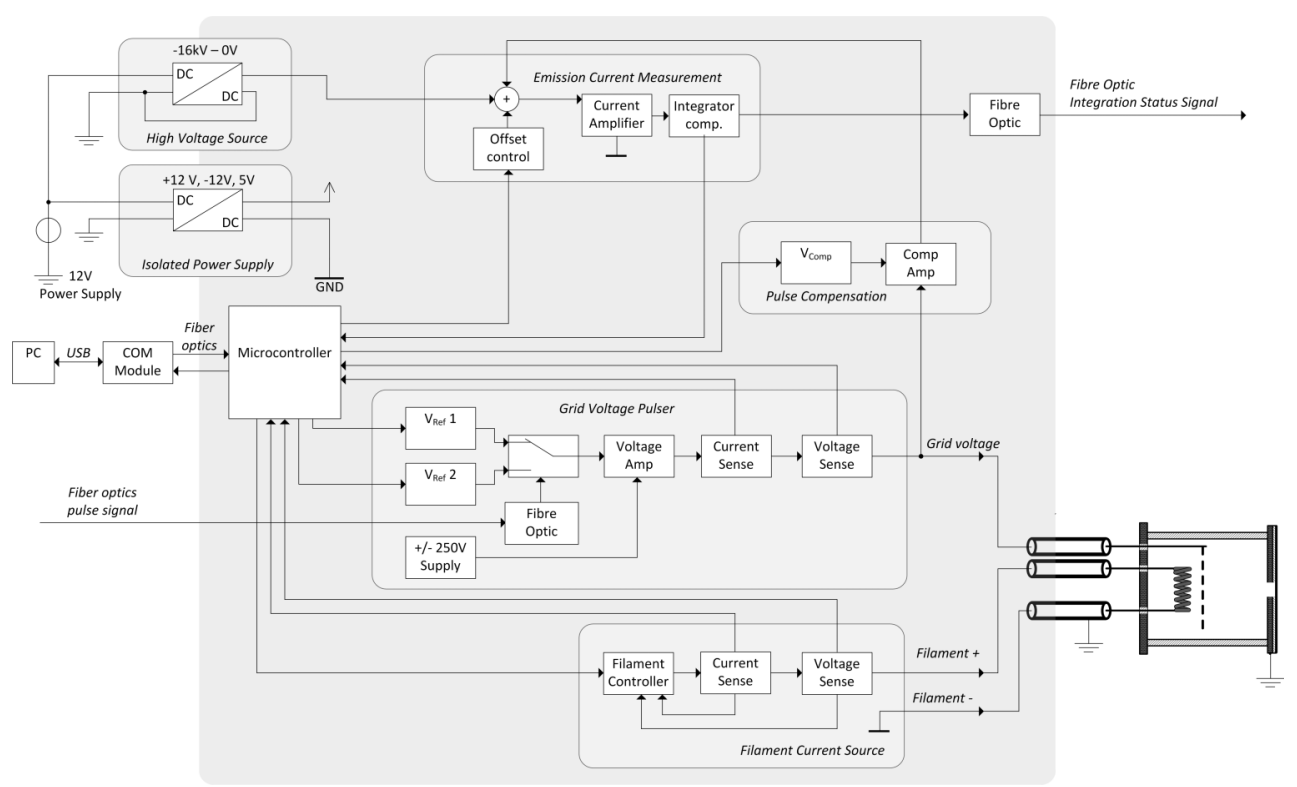

FIG. 4. Schematic setup of the control electronics. All blocks on the grey background are at the high voltage potential of up to $-16 \mathrm{kV}$.

as a change in voltage causes both capacitively coupled interferences and a change in the leakage current through the insulated power supply.

The "emission current measurement" consists of a transimpedance amplifier with its input connected to the high voltage source as shown on the left side of Figure 6. This is the only connection of other electronics to the high voltage source, as the transimpedance amplifier regulates its inputs in such a way that the ground potential of the high voltage electronics corresponds to the potential of the high voltage source. Thus, any current flowing from the high voltage potential to ground has to go through this amplifier and it therefore measures the current emitted by the tungsten filament that hits the acceleration electrode or passes through the $\mathrm{Si}_{3} \mathrm{~N}_{4}$ membrane, that is, the interior emission current. The current flowing back through the control grid is not measured, as it is only circulating within the high voltage electronics. This has the advantage that the relatively large grid current does not cause an offset in the measurement of the smaller interior emission current. However, the transimpedance amplifier circuit must also be thoroughly protected against overcurrents, which will not only occur during electrical breakdowns but also during a normal power-on. All the current to the high voltage potential, including the current required to charge all parasitic capacitances, flows through this amplifier. Thus, protection diodes and current limiting resistors are necessary at its input. The output signal of the transimpedance amplifier is digitized by a discrete 15-bit dual slope converter and passed to the microcontroller. As the conversion time of a dual slope converter is dependent on its input signal, an additional $1 \mathrm{G} \Omega$ resistor is connected to the input of the transimpedance amplifier. With the aid of this resistor, a correction current is injected into the input node, keeping the input signal of the dual slope converter always in the center of its range. Hence, the converter always has the maximum reserve for current transients occurring in both direction, regardless of the present emission current and leakage currents. Furthermore, the constant conversion time of the dual slope converter greatly simplifies the implementation of the digital controller. Using this measurement setup, an interior emission current resolution of about $300 \mathrm{fA}$ is achieved. This corresponds to an exterior emission current resolution of about $15 \mathrm{fA}$, as the exterior emission current observed at atmospheric pressure is dampened by a factor of about 20 as described above.

The "filament current source" is a voltage controlled current source that follows the output value of the digital controller. By adjusting the current through the filament, its temperature is regulated. It should be noted that the positive temperature coefficient of the tungsten filament's resistance would lead to an extremely high inrush current if it was operated at low temperatures using a voltage source instead of a current source. This should therefore be avoided in any case.

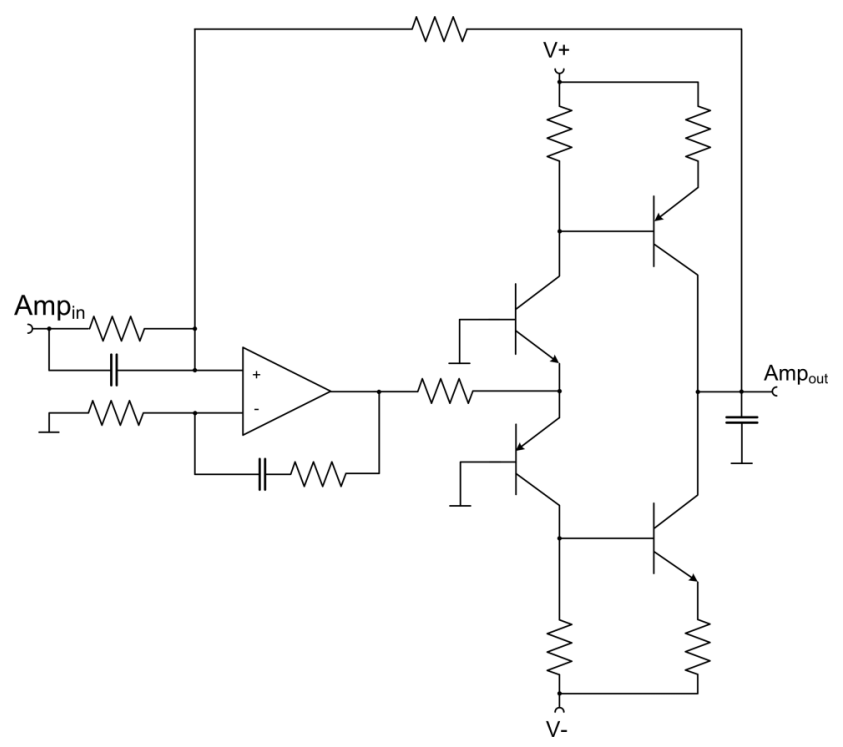

FIG. 5. Voltage amplifier inside the grid voltage pulser. 

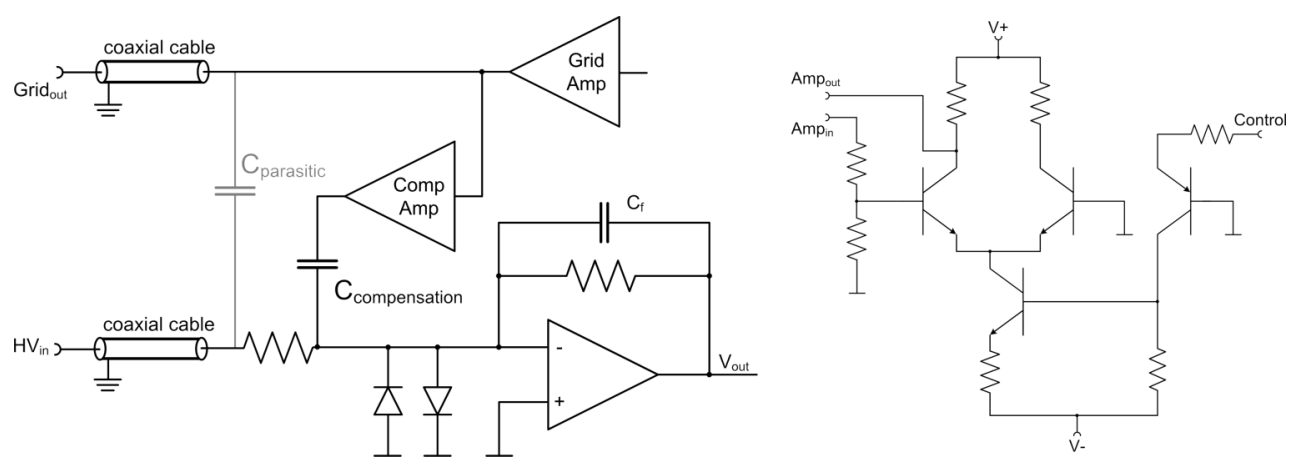

FIG. 6. Simplified schematic of the coupling paths of grid pulses including the compensation amplifier (left) and the compensation amplifier with voltage controlled gain (right).

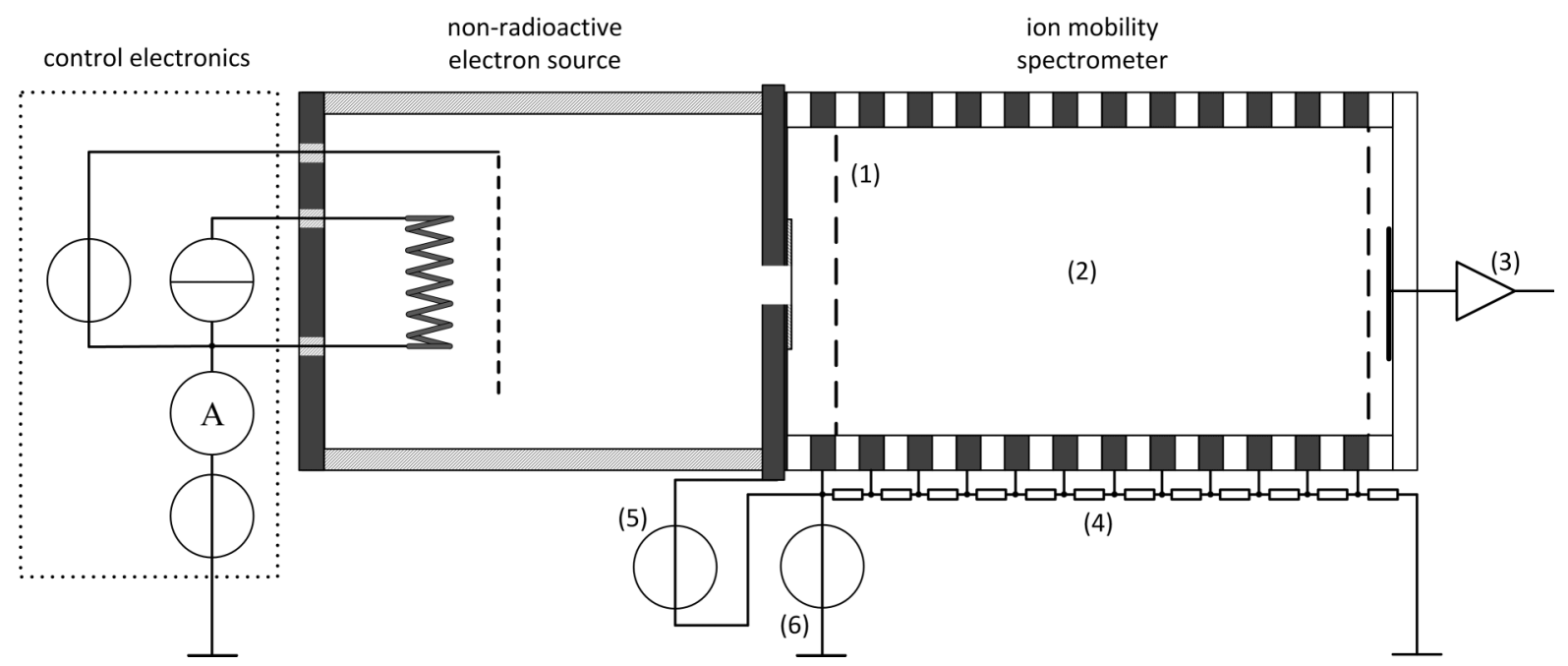

FIG. 7. Schematic setup of the non-radioactive electron source coupled to a ToF-IMS. (1) Ion injection grid, (2) ion drift region, (3) ion current amplifier, (4) voltage divider, (5) injection voltage source, (6) drift voltage source.

The "grid voltage pulser" provides the ability to switch the voltage at the control grid quickly in order to turn the emission on and off. For development purposes, a large voltage range is desirable, which is achieved by the bipolar current source shown in Figure 5. It utilizes voltage feedback to charge the control grid to the desired voltage. Supplied with $\pm 250 \mathrm{~V}$, it is able to switch the control grid between any two voltages in the range from $-240 \mathrm{~V}$ to $+240 \mathrm{~V}$ within a few microseconds. For example, the control grid of the electron source presented in this paper is typically switched between $-65 \mathrm{~V}$ and $-75 \mathrm{~V}$.

Last, the "pulse compensation amplifier" compensates errors in the current measurement arising from capacitive coupling while pulsing the control grid. For personal safety and electromagnetic compatibility reasons, all connections between the control electronics and the electron source need to be shielded high-voltage lines. As the shield is connected to ground, a significant capacitance between the control grid and ground results, which adds to any other existing parasitic capacitances. As a result, pulsing the control grid leads to a current flow through these capacitances to ground and then through the high voltage source. This current overdrives the regular current measurement, thus disturbing the emission current control loop. In order to minimize this interference current, a compensation amplifier is added to the setup

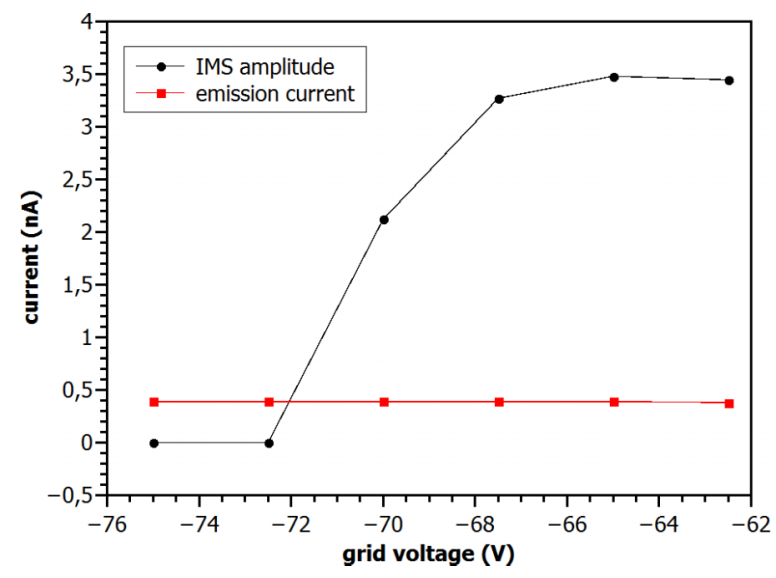

FIG. 8. Interior emission current (red squares) and ion current observed at atmospheric pressure (black circles) for different control grid voltages.

as shown on the left side of Figure 6. The voltage pulse applied to the grid is both scaled and phase-reversed by the compensation amplifier and then added to the input node of the current amplifier via a coupling capacitor. Ideally, the current picked up by the cable capacitance and the current through the coupling capacitor would interfere destructively, 

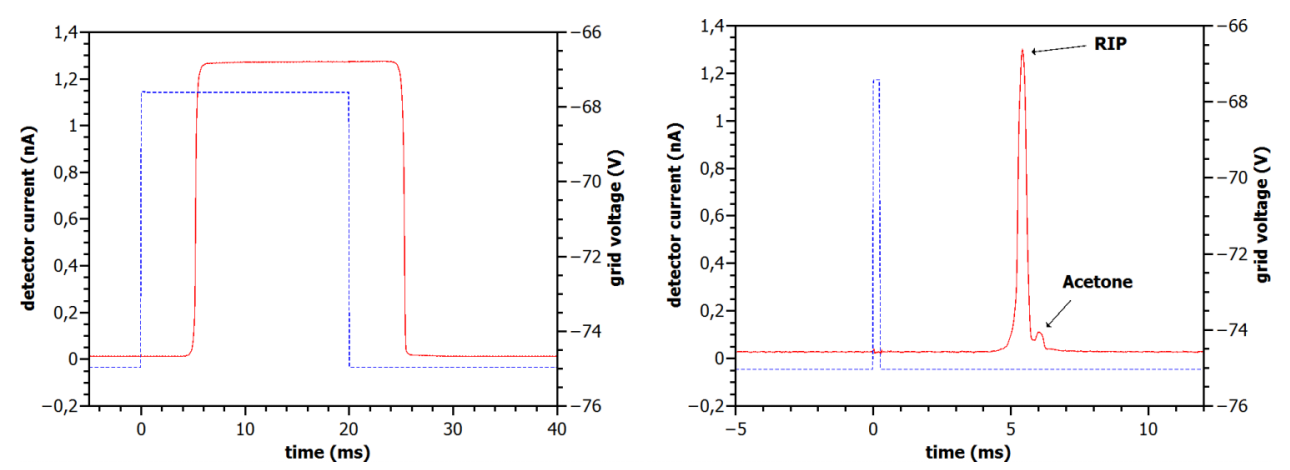

FIG. 9. Control grid voltages (dashed blue line) and resulting ion currents (solid red line) for emission pulses with a duration of $20 \mathrm{~ms}$ (left) and $100 \mu \mathrm{s}$ (right).

though it is not necessary to compensate the interference current completely as long as both pulse edges belong to one integration period, like in our setup. Thus, it is only necessary to ensure that neither the transimpedance amplifier nor the dual slope converter becomes overdriven. This is easily possible using the compensation amplifier and the offset correction mentioned previously.

As the cable capacitance depends on the cable length and different thermionic emitter setups may exhibit different parasitic capacitances, it is necessary to change the compensation amplifier's gain electronically. This was realized as shown on the right side of Figure 6, using two NPN transistors connected as a differential amplifier. The emitter current of the differential amplifier and hence the gain of this circuit can be adjusted by the microcontroller via the control input, allowing to tune the compensation amplifier for the best compensation possible.

\section{EXPERIMENTAL}

To verify the function of the electron source, it was coupled to a time-of-flight ion mobility spectrometer using dry clean air as drift gas as shown in Figure 7. The acceleration electrode was placed at the drift voltage potential of the IMS, which is $5 \mathrm{kV}$. Thus, to achieve the necessary kinetic energy of $10 \mathrm{keV}$, the electron source electronics and therefore the tungsten filament were placed at a potential of $-5 \mathrm{kV}$.

First, non-pulsed operation using the presented control electronics was characterized as shown in Figure 8, which displays both the ion current of the ion mobility spectrometer and the interior emission current for different control grid voltages. As the interior emission current remains constant for all control voltages of interest, it is assured that the thermionic emission is constantly operated in saturation, as demanded by the described operating principle. The ion current of the ion mobility spectrometer, and therefore the exterior emission current observed at atmospheric pressure, however is easily controlled by using different control grid voltages. As this voltage only needs to vary between $-65 \mathrm{~V}$ and $-75 \mathrm{~V}$ in order to switch the emission on and off, it is easily possible to pulse the electron beam very fast.

The use of the thermionic electron source as a replacement for a tritium source has already been examined in Refs. 18 and 16 showing comparable ion currents and identical ion chemistry. Therefore, we will only consider the pulsed operation in the following. Here, the ion shutter of the ion mobility spectrometer was set to be permanently open, thus allowing every positive ion created to move directly towards the detector. Then, short electron pulses were generated by the source and the resulting ion current was measured as shown in Figure 9.

First, a $20 \mathrm{~ms}$ emission pulse was generated while the ion mobility spectrometer was purged with clean air. The resulting ion current increases sharply with no overshoot and then remains constant for the duration of the emission pulse. The delay of the ion signal is $5.5 \mathrm{~ms}$, which corresponds to the time the ions generated from clean air, the so-called reactant ion peak (RIP), need to traverse our ion mobility spectrometer. Next, the emission pulse width was shortened to $100 \mu$ s and acetone was injected into the ionization region. This lead to the formation of an additional acetone ion species. Even though no ion shutter was used, both a reactant ion peak and an acetone ion peak are clearly visible and partly separated from each other, as the emission pulse is sufficiently short to create a defined ion plug.

\section{CONCLUSION}

In this paper, a design for a small, low-cost electron emitter with fast pulsing has been presented together with suitable control electronics. It utilizes a single control grid, whose potential needs to be varied by only $10 \mathrm{~V}$ in order to partially focus or defocus the electron beam. Only when the electron beam is focused, electrons are emitted outside the source, but the electron current inside the source can be measured continuously. This approach offers both greatly reduced instrumental effort and significantly improved emission current control compared to commercially available electron emitters. This is extremely important in order to obtain reproducible measurements, especially when studying ion chemistry. Furthermore, the ability to create short, stable emission pulses allows for the design and operation of a shutter-less ion mobility spectrometer, which would also be tremendously simpler than current designs.

${ }^{1}$ G. Eiceman and Z. Karpas, Ion Mobility Spectrometry (CRC Press, 2005).

${ }^{2}$ H. H. Hill, W. Siems, R. Louis, and D. McMinn, "Ion mobility spectrometry," Anal. Chem. 62, 1201A-1209A (1990).

${ }^{3}$ J. Langejuergen, T. Hopmeier, S. Meinen, E. Carstens, G. Theilmeier, W. Koppert, and S. Zimmermann, "Analysis of exhaled breath during surgery," in 14th International Meeting on Chemical Sensors-IMCS 2012 (IMCS, 2012), pp. 588-591. 
${ }^{4}$ A. Bruins, "Atmospheric-pressure-ionization mass spectrometry," TrAC, Trends Anal. Chem. 13(1), 37-43 (1994).

${ }^{5}$ H. Borsdorf and G. Eiceman, "Ion mobility spectrometry: Principles and applications," Appl. Spectrosc. Rev. 41, 323-375 (2006).

${ }^{6}$ S. R. Lipsky and J. E. Lovelock, "Electron affinity spectroscopy-A new method for the identification of functional groups in chemical compounds separated by gas chromatography," J. Am. Chem. Soc. 82, 431-433 (1960).

${ }^{7}$ R. J. Maggs, P. L. Joynes, A. J. Davies, and J. E. Lovelock, “The electron capture detector-A new mode of operation," Anal. Chem. 43, 1966-1971 (1971).

${ }^{8}$ B. Liu, D. Alvarez-Ossa, N. P. Kherani, S. Zukotynski, and K. P. Chen, "Gamma-free smoke and particle detector using tritiated foils," IEEE Sens. J. 7, 917-918 (2007).

${ }^{9}$ F. N. Simon and G. D. Rork, "Ionization-type smoke detectors," Rev. Sci. Instrum. 47, 74-80 (1976).

${ }^{10} \mathrm{P}$. Cochems, F. Gunzer, J. Langejuergen, A. Heptner, and S. Zimmermann, "Selective ion suppression as a pre-separation method in ion mobility spectrometry using a pulsed electron gun," Int. J. Ion Mobility Spectrom. 15, 31-39 (2011)

${ }^{11}$ A. Heptner, P. Cochems, J. Langejuergen, F. Gunzer, and S. Zimmermann, "Investigation of ion-ion-recombination at atmospheric pressure with a pulsed electron gun," Analyst 137, 5105-5112 (2012).
${ }^{12}$ F. T. Porter, "Beta decay energy of tritium," Phys. Rev. 115, 450-453 (1959).

${ }^{13}$ D. W. Hetherington, R. L. Graham, M. A. Lone, J. S. Geiger, and G. E. Lee-Whiting, "Upper limits on the mixing of heavy neutrinos in the beta decay of 63Ni," Phys. Rev. C 36, 1504-1513 (1987).

${ }^{14}$ W. B. Nottingham and G. Eastman, "Thermionic emission from tungsten and thoriated tungsten filaments," Phys. Rev. 49, 78-97 (1936).

${ }^{15} \mathrm{~F}$. Gunzer, A. Ulrich, and W. Baether, "A novel non-radioactive electron source for ion mobility spectrometry," Int. J. Ion Mobility Spectrom. 13, 9-16 (2010)

${ }^{16} \mathrm{P}$. Cochems, M. Runge, and S. Zimmermann, "A current controlled miniaturized non-radioactive electron emitter for atmospheric pressure chemical ionization based on thermionic emission," Sens. Actuators, A 206, 165-170 (2014).

${ }^{17}$ A. Morozov, T. Heindl, C. Skrobol, J. Wieser, R. Kruecken, and A. Ulrich, "Transmission of $\sim 10 \mathrm{keV}$ electron beams through thin ceramic foils: Measurements and Monte Carlo simulations of electron energy distribution functions," Eur. Phys. J. D 48, 383-388 (2008).

${ }^{18} \mathrm{P}$. Cochems, J. Langejuergen, A. Heptner, and S. Zimmermann, "Towards a miniaturized non-radioactive electron emitter with proximity focusing," Int. J. Ion Mobility Spectrom. 15, 223-229 (2012).

${ }^{19} \mathrm{G}$. L. Shen, "The pumping of methane by an ionization assisted $\mathrm{Zr} / \mathrm{Al}$ getter pump,” J. Vac. Sci. Technol., A 5(4), 2580-2583 (1986). 